\title{
Recognition of early-onset schizophrenia using deep-learning method
}

\author{
Shaoqiang Han' ${ }^{1}$, Wei Huang ${ }^{1}$, Yan Zhang ${ }^{2,3}$, Jingping Zhao ${ }^{2,3,4^{*}}$ and Huafu Chen ${ }^{\text {* }}$
}

\author{
*Correspondence: \\ zhaojingpinghunancsu@163. \\ com; chenhf@uestc.edu.cn \\ ${ }^{1}$ Center for Information \\ in BioMedicine, \\ Key Laboratory \\ for Neuroinformation \\ of Ministry of Education, \\ School of Life Science \\ and Technology, University \\ of Electronic Science \\ and Technology of China, \\ Chengdu 610054, China \\ ${ }^{2}$ Mental Health Institute, \\ Key Laboratory for Mental \\ Health of Hunan Province, \\ The Second Xiangya Hospital \\ of Central South University, \\ 139, Middle Renmin Road, \\ Changsha 410011, Hunan, \\ China \\ Full list of author information \\ is available at the end of the \\ article
}

\begin{abstract}
Functional connectivity at resting state was found altered in early-onset schizophrenia patients. However, its potential as biomarker of clinical diagnosis is unknown. To test whether resting-state functional connectivity can be a potential biomarker of classifying patients from controls, 39 early-onset schizophrenia patients and 31 healthy controls were included in our study. Resting-state functional MRI networks were built with the whole brain atlas as classification features to distinguish patients from healthy controls. Three-stage deep-learning network was used to deduce dimension reduction, and feedforward back propagation neural networks were used as classifier. As the result, the classification accuracy reached $79.3 \%$ (87.4\% for sensitivity, $82.2 \%$ for specificity, $p<0.05$ for permuted test). Our works showed us that resting-state connectivity presented good potential classification capacity and can be used as biomarker of clinical diagnosis.
\end{abstract}

Keywords: Functional connectivity, Pattern recognition, Deep-learning

\section{Introduction}

Schizophrenia is a complex syndrome lacking integration between thought, emotion, and behavior cognitive and affective deficits (Fornito et al. 2012). The disease affects nearly $1 \%$ of the world's population (McGrath et al. 2008). Early diagnosis can significantly improve treatment response and reduce associated costs (McGlashan 1998). The absence of stable and reliable biomarkers makes current diagnosis of schizophrenia, which mainly relies on clinical manifestations by experienced clinician, a challenging task (Nieuwenhuis et al. 2012). In recent years, fMRI has turned out a powerful tool to study psychosis. Especially, resting-state functional connectivity, in the absence of explicit task, captures a spontaneous, stable, and intrinsic property of brain functional organization (Shehzad et al. 2009; Fox and Raichle 2007). Dysfunctional connectivity in resting state was found in schizophrenia patients (Woodward et al. 2011; WhitfieldGabrieli et al. 2009; Rotarska-Jagiela et al. 2010). Whether resting-state functional connectivity has potential to be diagnostic indicators is unknown.

Small number of subjects and much too higher-dimensional data in fMRI make it a challenge to design an accurate, robust classifier to discriminate and research schizophrenia. To address this critical issue, different types of feature extraction, selection methods have been proposed to reduce the dimensionality of feature space. However,

(c) The Author(s) 2017. This article is distributed under the terms of the Creative Commons Attribution 4.0 International License (http://creativecommons.org/licenses/by/4.0/), which permits unrestricted use, distribution, and reproduction in any medium, provided you give appropriate credit to the original author(s) and the source, provide a link to the Creative Commons license, and indicate if changes were made. 
traditional feature selection or extraction methods mainly rely too much on mathematical method and ignore physiological signification; those features that are discarded mathematically may have vital positions in emergence and development of disease. Making an accurate prediction with high-dimensional data seems a difficult job (Liu et al. 2012). In recent years, deep-learning turns out to be a good solution to this problem (Hinton and Salakhutdinov 2006). By applying autoencoder method, high-dimensional data were mapped in low dimension, this method presented good practicability (Hazlett et al. 2017). The model used in our research was first raised by Hinton and Salakhutdinov (2006); its core idea is that high-dimensional data can be converted to low-dimensional codes by training a multilayer neural networks with a small central layer to reconstruct high-dimensional input vectors. Comparing with most used PCA, this model shows a distinct advantage.

In current study, resting state was calculated and used as classification features and sent to Three-stage deep-learning network. Then feedforward back propagation neural networks were used as classifier to classify patients from controls. We expected to find that resting-state functional connectivity presented good potential classification capacity.

\section{Materials and methods Participants}

A total of 39 EOS patients were recruited from the Second Affiliated Hospital of Xinxiang Medical University. All patients were independently diagnosed by research psychiatrists and satisfied the following criteria: (1) DSM-IV-TR criteria for schizophrenia (Diagnostic and Statistical Manual of Mental Disorders, fourth edition, text revision, American Psychiatric Association, 2000); (2) no co-morbid Axis I diagnosis; (3) duration of illness is less than 2 years; (4) anti-psychotic-naive. Patients were interviewed again after 6 months for a final schizophrenia diagnosis. The symptoms were evaluated using the Positive and Negative Syndrome Scale (PANSS). A total of 31 age-, gender-, education-, and IQ-matched healthy adolescents were included in this study. All HCs did not have any past or current neurological disorders, family history of hereditary neurological disorders, and history of head injury resulting in loss of consciousness and substance abuse.

This study was approved by the Ethics Committee of the Second Affiliated Hospital of Xinxiang Medical University, and informed written consents were obtained from all subjects.

\section{Data acquisition}

All subjects were instructed to rest with their eyes closed, not to think of anything in particular, and not to fall asleep during the resting-state fMRI (rs-fMRI)scanning(Marx et al. 2004; Pang et al. 2015; Wei et al. 2014). fMRI data were collected using the 3T MRI scanner (Siemens-Trio, Erlangen, Germany) of the Second Affiliated Hospital of Xinxiang Medical University. Scanning and clinical assessments were performed in 1 day. Functional images were collected transversely using an echo-planar imaging (EPI) sequence with the following settings: TR/TE $=2000 / 30 \mathrm{~ms}$, flip angle $=90^{\circ}$, FOV $=220 \times 220 \mathrm{~mm}^{2}$, slices $=33$, matrix $=64 \times 64$, inter slice gap $=0.6 \mathrm{~mm}$, and 
voxel size $=3.44 \times 3.44 \times 4 \mathrm{~mm}^{3}$. The scan lasted for $480 \mathrm{~s}$ for each subject, and 240 volumes were acquired.

\section{Image preprocessing}

Data preprocessing was conducted using the Statistical Parametric Mapping Software (SPM8, http://www.fil.ion.ucl.ac.uk/spm8). The first 10 volumes of each participant were discarded because of the instability of the initial MRI signal and adaptation of the participants to the circumstance, leaving 230 volumes. The remaining rs-fMRI images were corrected for slice acquisition and head motion using a least squares approach with a 6-parameter spatial transformation. Four patients and one healthy control with head motion scans exceeding $2 \mathrm{~mm}$ or $1^{\circ}$ rotation were excluded. Subsequently, the corrected images were normalized according to the standard SPM8 Montreal Neurological Institute (MNI) template (Power et al. 2012) and re-sampled to $3 \times 3 \times 3 \mathrm{~mm}^{3}$ voxel size. The resulting images were linearly detrended and filtered using a typical temporal band-pass, including slow- 5 band-pass $(0.01-0.027 \mathrm{~Hz})$ and slow-4 band-pass $(0.027-0.073 \mathrm{~Hz})$ separately (Yu et al. 2014; Gohel et al. 2014). Friston 24 motion parameters, cerebrospinal fluid, and white matter signals were included in the multiple regression model to reduce the effects of head motion and non-neuronal BOLD fluctuations (Friston et al. 1994; Tomasi and Volkow 2012). Given that resting-state FCD is sensitive to minor head movements, we calculated the mean frame-wise displacement (FD) to further determine the comparability of head movement across groups. The largest mean FD of each subjects was less than $0.3 \mathrm{~mm}$ and two-sample $t$ test showed that there was no significant difference in the mean FD between the two groups (HC: $0.09 \pm 0.05$; EOS $0.10 \pm 0.03$; mean $\pm \mathrm{SD}, p=0.33$ ). Then, the "bad" time points as well as their 1-back and 2-forward time points were removed from the time series by employing a "scrubbing" method with a FD (Power et al. 2012; Long et al. 2016) threshold of $0.5 \mathrm{~mm}$. Participants retaining more than $80 \%$ of the original signals after scrubbing were included in the analysis. The number of time points that remained was non-significantly different between HC $(227.8 \pm 6.98)$ and EOS patients $(225.1 \pm 6.72)(p=0.09)$.

\section{Extraction of regional time series and construction of functional connectivity network}

Images were smoothed using an $8 \times 8 \times 8 \mathrm{~mm}^{3} \mathrm{FWHM}$ Gaussian kernel. The averaged signals of 90 brain regions of the AAL (excluding brain areas in the cerebellum) template were calculated. We then computed the Pearson's correlation between each pair of signals. Thus, a $90 \times 90$ connectivity matrix for each subject was obtained.

\section{Deep-learning analysis}

Functional connectivity was treated as classification features and sent to autoencoder. As done in previous studies (Hinton and Salakhutdinov 2006; Hazlett et al. 2017), the model was evaluated via a standard tenfold cross-validation. The core of model was a weighted three-stage neural/deep-learning network, where the first stage reduces 4005 measurements to 400 , the second stage reduces 400 to 20 , and the third stage reduces 20 to only 2 measurements. At each stage, the measurements (in the progressively smaller sets) are the weighted combination of input measurements from the previous stage (Fig. 1). 


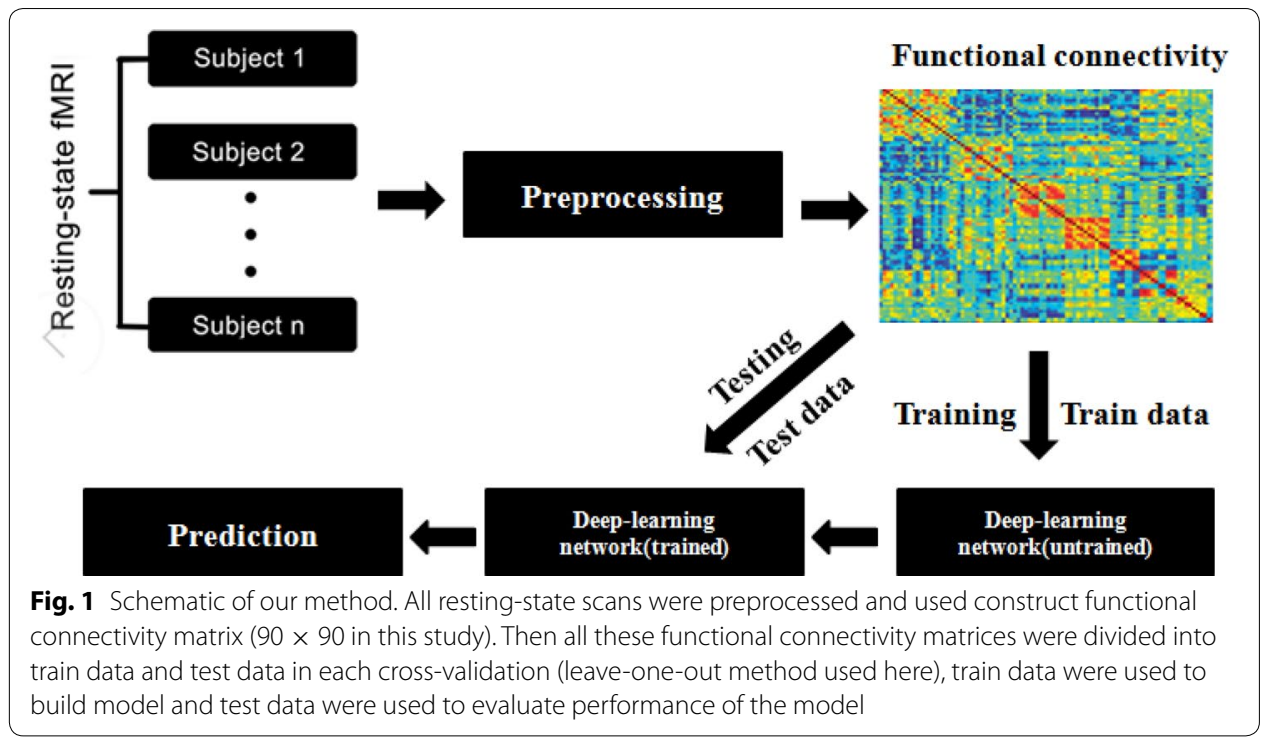

\section{Results}

\section{Demographic information}

Sample characteristics are shown in Table 1. No significant difference was found between schizophrenias and controls in sociodemographic characteristics like age, gender, and so on.

\section{Performance of deep-learning}

The accuracy of deep-learning method reached $79.3 \%$, the sensitivity reached $87.4 \%$, and the specificity reached $82.2 \%$. Then, to evaluate the significance of the accuracy, permuted test was the used. By permuting 10,000 times, the $p$ value was smaller than 0.05 , this result showed us that the accuracy of deep-learning was significant (Table 2).

\section{Table 1 Demographic and clinical characteristics in the study}

\begin{tabular}{llll}
\hline Demographics, mean (SD) & $\begin{array}{l}\text { EOS } \\
\boldsymbol{n = 3 7}\end{array}$ & $\begin{array}{l}\text { Control } \\
\boldsymbol{n}=\mathbf{3 0}\end{array}$ & $\boldsymbol{p}$ value \\
\hline Age (year) & $15.5(1.8)$ & $15.3(1.6)$ & $0.57^{\mathrm{a}}$ \\
Gender (male/female) & $20 / 17$ & $13 / 17$ & $0.53^{\mathrm{b}}$ \\
Education (years) & $8.5(1.48)$ & $8.7(1.42)$ & $0.605^{\mathrm{a}}$ \\
Duration of psychosis (months) & $16.0(14.4)$ & - & - \\
Handedness (right/left) & $37 / 0$ & - & - \\
PANSS positive symptoms & $20.42(5.72)$ & - & - \\
PANSS negative symptoms & $20.91(8.41)$ & - & - \\
PANSS general symptoms & $33.28(6.69)$ & - & - \\
PANSS total symptoms & $74.62(10.61)$ & & - \\
\hline
\end{tabular}

a $p$ value was obtained by two-sample $t$ test

b $p$ value was obtained by $x^{2}$ two-tailed test

Table 2 Performance of deep-learning method

\begin{tabular}{llll}
\hline Classification & Sensitivity & Specificity & $\boldsymbol{p}$ value (permuted) \\
\hline $79.3 \%$ & $87.4 \%$ & $82.2 \%$ & $<0.05$ \\
\hline
\end{tabular}




\title{
Discussion
}

In current study, we used deep-learning method to verify the potential of functional connectivity in resting state used as biomarker of clinical diagnosis. Resting-state connectivity presented good potential classification capacity (79.3\% for classification accuracy, $87.4 \%$ for sensitivity, $82.2 \%$ for specificity, $p<0.05$ for permuted test).

Many other studies used resting-state functional connectivity to differentiate schizophrenia patients from controls (Mikolas et al. 2016; Cabral et al. 2016; Arbabshirani et al. 2013; Shen et al. 2010; Du et al. 2012; Skåtun et al. 2016). Two strategies were used in these studies to overcome over-fitting problem. First, feature selection/extraction method was used to reduce the dimensionality of feature space, then selected features were used to classify patients with schizophrenia from controls. Second, focusing on feature subsets and test their ability of classification. The first strategy rely too much on mathematical method and ignore physiological signification; those features that are discarded mathematically may have vital positions in emergence and development of disease (Liu et al. 2012). As for the second strategy, focusing on partial feature subsets would lead to a biased conclusion (Dietterich 1997). Deep-learning used in our study has been proved to be a good solution to these problems (Hinton and Salakhutdinov 2006). Here, we just proved that deep-learning method could be a feasible tool to recognize schizophrenia patients from controls.

Early diagnosis can significantly improve treatment response and reduce associated costs (McGlashan 1998). The absence of stable and reliable biomarkers makes current diagnosis of schizophrenia, which mainly relies on clinical manifestations by experienced clinician for now, a challenging task. Previous studies had found that resting-state functional connectivity was altered in schizophrenia patients (Woodward et al. 2011; Whitfield-Gabrieli et al. 2009; Rotarska-Jagiela et al. 2010). Our study found that restingstate functional connectivity presented good potential classification capacity and could be used as a biomarker of clinical diagnosis.

\section{Authors' contributions}

JZ conceived and designed the study. YZ collected the clinical data. SH and WH analyzed the data and also wrote the paper. All authors read and approved the final manuscript.

\begin{abstract}
Author details
${ }^{1}$ Center for Information in BioMedicine, Key Laboratory for Neuroinformation of Ministry of Education, School of Life Science and Technology, University of Electronic Science and Technology of China, Chengdu 610054, China. ${ }^{2}$ Mental Health Institute, Key Laboratory for Mental Health of Hunan Province, The Second Xiangya Hospital of Central South University, 139, Middle Renmin Road, Changsha 410011, Hunan, China. ${ }^{3}$ Department of Psychiatry, The Second Affiliated Hospital of Xinxiang Medical University, Xinxiang, China. ${ }^{4}$ Mental Health Institute, The Second Xiangya Hospital of Central South University, 139, Middle Renmin Road, Changsha 410011, Hunan, China.
\end{abstract}

\section{Acknowledgements}

The authors thank the patients and volunteers for participating in this study.

Competing interests

The authors declare that they have no competing interests.

\section{Availability of data and materials}

None.

\section{Ethics approval and consent to participate}

This study was approved by the Ethics Committee of the Second Affiliated Hospital of Xinxiang Medical University, and informed written consents were obtained from all subjects.

\section{Funding}

This research was supported by the 863 project (2015AA020505), the Natural Science Foundation of China (61533006 and 61673089), the project of the Science and Technology Department in Sichuan province (2017JY0094), and the Fundamental Research Funds for Central Universities (ZYGX2016KYQD120 and ZYGX2015J141). 


\section{Publisher's Note}

Springer Nature remains neutral with regard to jurisdictional claims in published maps and institutional affiliations.

Received: 30 October 2017 Accepted: 23 November 2017

Published online: 06 December 2017

\section{References}

Arbabshirani MR et al (2013) Classification of schizophrenia patients based on resting-state functional network connectivity. Front Neurosci 7:16

Cabral C et al (2016) Classifying schizophrenia using multimodal multivariate pattern recognition analysis: evaluating the impact of individual clinical profiles on the neurodiagnostic performance. Schizophr Bull 42:S110-S117

Dietterich TG (1997) Machine-learning research_four current directions. Ai Magazine 18(4):97-136

Du W et al (2012) High classification accuracy for schizophrenia with rest and task fMRI data. Front Hum Neurosci 6:12

Fornito A et al (2012) Schizophrenia, neuroimaging and connectomics. Neuroimage 62(4):2296-2314

Fox MD, Raichle ME (2007) Spontaneous fluctuations in brain activity observed with functional magnetic resonance imaging. Nat Rev Neurosci 8(9):700-711

Friston KJ et al (1994) Statistical parametric maps in functional imaging: a general linear approach. Hum Brain Mapp 2(4):189-210

Gohel SR, Biswal BB (2015) Functional integration between brain regions at rest occurs in multiple-frequency bands. Brain Connect 5(1):23-34

Hazlett HC et al (2017) Early brain development in infants at high risk for autism spectrum disorder. Nature 542(7641):348

Hinton GE, Salakhutdinov RR (2006) Reducing the dimensionality of data with neural networks. Science 313(5786):504

Liu MH et al (2012) Ensemble sparse classification of Alzheimer's disease. Neuroimage 60(2):1106-1116

Long Z et al (2016) Alteration of functional connectivity in autism spectrum disorder: effect of age and anatomical distance. Sci Rep 6:26527

Marx E et al (2004) Eyes open and eyes closed as rest conditions: impact on brain activation patterns. Neuroimage 21(4):1818-1824

McGlashan TH (1998) Early detection and intervention of schizophrenia: rationale and research. Br J Psychiatry 172:3-6

McGrath J et al (2008) Schizophrenia: a concise overview of incidence, prevalence, and mortality. Epidemiol Rev 30(1):67-76

Mikolas P et al (2016) Connectivity of the anterior insula differentiates participants with first-episode schizophrenia spectrum disorders from controls: a machine-learning study. Psychol Med 46(13):2695-2704

Nieuwenhuis M et al (2012) Classification of schizophrenia patients and healthy controls from structural MRI scans in two large independent samples. Neuroimage 61(3):606-612

Pang Y, Cui Q, Duan X et al (2015) Extraversion modulates functional connectivity hubs of resting-state brain networks. J Neuropsychol 11(3):347-361. https://doi.org/10.1111/jnp.12090

Power JD et al (2012) Spurious but systematic correlations in functional connectivity MRI networks arise from subject motion. Neuroimage 59(3):2142-2154

Rotarska-Jagiela A et al (2010) Resting-state functional network correlates of psychotic symptoms in schizophrenia. Schizophr Res 117(1):21-30

Shehzad Z et al (2009) The resting brain: unconstrained yet reliable. Cereb Cortex 19(10):2209

Shen $\mathrm{H}$ et al (2010) Discriminative analysis of resting-state functional connectivity patterns of schizophrenia using low dimensional embedding of fMRI. Neuroimage 49(4):3110-3121

Skåtun KC et al (2016) Consistent functional connectivity alterations in schizophrenia spectrum disorder: a Multisite Study. Schizophr Bull 43(4):914-924

Tomasi D, Volkow ND (2012) Aging and functional brain networks. Mol Psychiatry 17(5):549-558

Wei $L$ et al (2014) Specific frequency bands of amplitude low-frequency oscillation encodes personality. Hum Brain Mapp 35(1):331-339

Whitfield-Gabrieli S et al (2009) Hyperactivity and hyperconnectivity of the default network in schizophrenia and in firstdegree relatives of persons with schizophrenia. Proc Natl Acad Sci USA 106(4):1279-1284

Woodward ND, Rogers B, Heckers S (2011) Functional resting-state networks are differentially affected in schizophrenia. Schizophr Res 130(1-3):86-93

Yu R et al (2014) Frequency-specific alternations in the amplitude of low-frequency fluctuations in schizophrenia. Hum Brain Mapp 35(2):627-637 